\title{
Further Promotion of Quadratic Time-Varying Parameters Discrete Grey Model
}

\author{
Mengdi Jin*, Jiwei Liu \\ School of Science, Communication University of China, Beijing, China \\ Email address: \\ jmdacr@163.com (Mengdi Jin), xunchanglu0901@163.com (Jiwei Liu) \\ ${ }^{*}$ Corresponding author
}

To cite this article:

Mengdi Jin, Jiwei Liu. Further Promotion of Quadratic Time-Varying Parameters Discrete Grey Model. American Journal of Information Science and Technology. Vol. 2, No. 3, 2018, pp. 74-82. doi: 10.11648/j.ajist.20180203.12

Received: October 23, 2018; Accepted: November 14, 2018; Published: December 19, 2018

\begin{abstract}
Based on the reason that the traditional buffer operator cannot adjust the action intensity, this paper proposes a positive real order weakening buffer operator, which solves the disadvantage that the original operator cannot be fine-tuned, and is more suitable for real life systems. By defining positive real order weakening buffer operator and according to the combination number and the nature of gamma function, the two are connected, and the positive real order weakening buffer sequence is transformed by gamma function. Next a quadratic time-varying linear parameter grey discrete prediction model (QTDGM) is established by using the constructed positive real order weakening buffer operator. The iterative optimization method of simulation base value is given, and the optimization model is established and the solution algorithm is proposed. Finally, the steps of modeling and forecasting by using QDGM model are described. In the case of science popularization fund forecast and raw coal output forecast, QTDGM model shows superior prediction effect. The relative error of the model is $0.34 \% \sim 7 \%$ in the three cases, which is much lower than that of the model using integer order weakening buffer operator and also lower than that of the linear time-varying parameter grey discrete model. QTDGM is more suitable for complex sample systems.
\end{abstract}

Keywords: Grey System, Fractional Order Buffer Operator, Qtdgm, Iteration and Optimization

\section{Introduction}

In fact, data prediction is to infer the law of development of things from the observed data, and then predict and estimate the unknown data according to the inferred law. The key is to effectively infer the law of development of things. Model-based forecasting method is to estimate the parameters of the model by establishing a parametric model matching the development of things and using the existing nonnegative observation data, so as to obtain the law of things' development. In order to improve the reliability and accuracy of prediction, the first condition is to select a good prediction model. Excellent prediction model means that the development law and matching between the model and things, as well as the number of model parameters are small. Less parameter model is conducive to reducing errors and improving prediction accuracy.

From the point of view of prediction error, besides choosing a good prediction model, the model-based prediction method will also face two problems in the process of dealing with the actual system: Firstly, due to the limitation of actual conditions, the observed data cannot accurately map with the actual data, and there are often uncertain disturbance components, that is, the observed data is noisy; The law of development of things is not constant, it is usually a slow process, that is to say, the parameters of the model are time-varying. From the point of view of the realization of model parameter estimation, the two problems are contradictory: Because the parameters of the model are time-varying, the weight of the parameters should be concentrated on the observation values closest to the predicted data as far as possible, so that the parameters of the model can be matched quickly and the errors caused by the changes of the parameters can be reduced. On the other hand, from the point of view of weakening the uncertain impact disturbance, it is necessary to spread the weights of parameter estimation to different observation values as far as possible. Then the smoothing method is used to reduce the prediction error caused by disturbance components.

With the growing demand for reliable small sample statistics, small sample prediction becomes a very important 
topic. Over the years, scholars have done in-depth research on the prediction of small sample non-stationary time series. The prominent problem is that, for non-stationary time series, when the system is seriously affected by disturbances, the available data in the past cannot truly reflect the law of the system. Therefore, in order to reduce the disturbance bound of the prediction model, positive real order weakening buffer operator is introduced to fine-tune the original sequence to improve the prediction accuracy.

As a novel prediction method, grey system [1] uses accumulation operator to improve the matching degree between data sequence and selected model [2], and uses buffer operator to reduce the impact of disturbance. By choosing a good model with few parameters, the grey system can effectively predict the system with few data and poor information. Therefore, in dealing with disturbances, the grey system allocates the weight of parameter estimation to the observed data by choosing different buffer operator, i.e. different weight allocation schemes.

On the basis of document [3-5], a discrete grey model with quadratic time-varying parameters is established by introducing a weakening buffer operator of positive real order. Case studies show that the model has good simulation and prediction accuracy. By comparing the prediction results of positive real order and integer order operator, the model established by positive real order weakening buffer operator can reasonably distribute the weights of each nonnegative observation data, so it can achieve more accurate parameter estimation. The positive real order weakening buffer operator is more suitable for complex real systems.

\section{The Construction of Positive Real Order Weakening Buffer Operator}

The classical weakening buffer operator fully considers the priority of each data, while the variable weight weakening buffer operator only considers the priority of the latest data. Therefore, considering the comprehensive utilization of the original data information, the classical weakening buffer operator is a good buffer operator. It is easy to prove that the higher the order of the classical weakening buffer operator, the better the effect of new information and the better the prediction quality. However, the classical weakening buffer operator cannot achieve the fine-tuning of the buffer effect intensity. In this paper, the positive real order weakening buffer operator is introduced.

Lemma1 $X^{(0)}$ is the nonnegative observation data sequence. The matrix of the 1 order weakening buffer operator $D$ is:

$$
X^{(0)} D=\left[\begin{array}{cccc}
\frac{1}{n} & \frac{1}{n} & \cdots & \frac{1}{n} \\
0 & \frac{1}{n-1} & \cdots & \frac{1}{n-1} \\
\cdots & \cdots & \cdots & \cdots \\
& & \cdots & 1
\end{array}\right]\left[\begin{array}{c}
x^{(0)}(1) \\
x^{(0)}(2) \\
\cdots \\
x^{(0)}(n)
\end{array}\right]=A\left(x^{(0)}\right)^{T} .
$$

Where $A$ is the matrix form of $D$. The matrix form of the two order weakening buffer operator $\mathrm{D}$ is:

$$
X^{(0)} D^{2}=\left[\begin{array}{cccc}
\frac{1}{n} & \frac{1}{n} & \cdots & \frac{1}{n} \\
0 & \frac{1}{n-1} & \cdots & \frac{1}{n-1} \\
\cdots & \cdots & \cdots & \cdots \\
& & \cdots & 1
\end{array}\right]^{2}\left[\begin{array}{c}
x^{(0)}(1) \\
x^{(0)}(2) \\
\cdots \\
x^{(0)}(n)
\end{array}\right]=A^{2}\left(X^{(0)}\right)^{T}
$$

Then $r\left(r \in N^{+}\right)$order weakening buffer operator:

$$
X^{(0)} D^{r}=\left[\begin{array}{cccc}
\frac{1}{n} & \frac{1}{n} & \cdots & \frac{1}{n} \\
0 & \frac{1}{n-1} & \cdots & \frac{1}{n-1} \\
\cdots & \cdots & \cdots & \cdots \\
& & \cdots & 1
\end{array}\right]^{r}\left[\begin{array}{c}
x^{(0)}(1) \\
x^{(0)}(2) \\
\cdots \\
x^{(0)}(n)
\end{array}\right]=A^{r}\left(X^{(0)}\right)^{T}
$$

The bigger the $r$ the weaker the effect of the buffer operator is, the more powerful the new information is. The following theorem shows that when $r<0$, the original formula becomes an reinforcement buffer operator.

Theorem 1 When $r<0$,

$$
X^{(0)} D^{r}=\left[\begin{array}{cccc}
\frac{1}{n} & \frac{1}{n} & \cdots & \frac{1}{n} \\
0 & \frac{1}{n-1} & \cdots & \frac{1}{n-1} \\
\cdots & \cdots & \cdots & \cdots \\
& & \cdots & 1
\end{array}\right]^{r}\left[\begin{array}{c}
x^{(0)}(1) \\
x^{(0)}(2) \\
\cdots \\
x^{(0)}(n)
\end{array}\right]=A^{r}\left(X^{(0)}\right)^{T}
$$

$D$ is the reinforcement buffer operator.

Proof when $r=-1$,

$$
\begin{aligned}
X^{(0)} D^{-1} & =\left[\begin{array}{cccc}
\frac{1}{n} & \frac{1}{n} & \cdots & \frac{1}{n} \\
0 & \frac{1}{n-1} & \cdots & \frac{1}{n-1} \\
\cdots & \cdots & \cdots & \cdots \\
\cdots & 1
\end{array}\right]^{-1}\left[\begin{array}{c}
x^{(0)}(1) \\
x^{(0)}(2) \\
\cdots \\
x^{(0)}(n)
\end{array}\right]= \\
A^{-1}\left(X^{(0)}\right)^{T} & =\left[\begin{array}{ccccc}
n & -(n-1) & 0 & \cdots & 0 \\
0 & n-1 & -(n-2) & \cdots & 0 \\
\cdots & \cdots & \cdots & \cdots & \cdots \\
0 & 0 & 0 & \cdots &
\end{array}\right]\left[\begin{array}{c}
x^{(0)}(1) \\
x^{(0)}(2) \\
\cdots \\
x^{(0)}(n)
\end{array}\right]
\end{aligned}
$$

If nonnegative observation data sequence $X^{(0)}$ is a monotonic attenuation sequence, according to $A\left(X^{(0)}\right)^{T} \leq\left(X^{(0)}\right)^{T}, A$ is a reversible matrix. Therefore $A^{-1} A\left(X^{(0)}\right)^{T} \leq A^{-1}\left(X^{(0)}\right)^{T}$, and $\left(X^{(0)}\right)^{T} \leq A^{-1}\left(X^{(0)}\right)^{T}$, i.e. $D^{-1}$ is an reinforcement buffer operator for monotonic attenuation sequences. For the same reason, $D^{-1}$ is an 
reinforcement buffer operator for monotonically increasing sequences.

If $X^{(0)}$ is the oscillation sequence, let

$$
\begin{aligned}
& x^{(0)}(l)=\max \left\{x^{(0)}(k), k=1,2, \cdots n\right\}, \\
& x^{(0)}(h)=\min \left\{x^{(0)}(k), k=1,2, \cdots n\right\} .
\end{aligned}
$$

$$
A\left[\begin{array}{c}
x^{(0)}(l) \\
x^{(0)}(l) \\
\cdots \\
x^{(0)}(l)
\end{array}\right] \leq\left[\begin{array}{c}
x^{(0)}(l) \\
x^{(0)}(l) \\
\cdots \\
x^{(0)}(l)
\end{array}\right], \quad A \quad \text { is } \quad \text { a } \quad \text { reversible matrix. }
$$$$
A^{-1} A\left[\begin{array}{c}
x^{(0)}(l) \\
x^{(0)}(l) \\
\ldots \\
x^{(0)}(l)
\end{array}\right] \leq A^{-1}\left[\begin{array}{c}
x^{(0)}(l) \\
x^{(0)}(l) \\
\ldots \\
x^{(0)}(l)
\end{array}\right], \text { and }\left[\begin{array}{c}
x^{(0)}(l) \\
x^{(0)}(l) \\
\ldots \\
x^{(0)}(l)
\end{array}\right] \leq A^{-1}\left[\begin{array}{c}
x^{(0)}(l) \\
x^{(0)}(l) \\
\ldots \\
x^{(0)}(l)
\end{array}\right] \text {. }
$$

For the same reason, $\left[\begin{array}{c}x^{(0)}(h) \\ x^{(0)}(h) \\ \ldots \\ x^{(0)}(h)\end{array}\right] \geq A^{-1}\left[\begin{array}{c}x^{(0)}(h) \\ x^{(0)}(h) \\ \ldots \\ x^{(0)}(h)\end{array}\right]$, therefore

$D^{-1}$ is the enhanced buffer operator of the oscillation sequence.

For the same reason, when $r<0$,

$$
X^{(0)} D^{r}=\left[\begin{array}{cccc}
\frac{1}{n} & \frac{1}{n} & \cdots & \frac{1}{n} \\
0 & \frac{1}{n-1} & \cdots & \frac{1}{n-1} \\
\cdots & \cdots & \cdots & \cdots \\
& & \cdots & 1
\end{array}\right]^{r}\left[\begin{array}{c}
x^{(0)}(1) \\
x^{(0)}(2) \\
\cdots \\
x^{(0)}(n)
\end{array}\right]=A^{r}\left(X^{(0)}\right)^{T}
$$

$D$ is an reinforcement buffer operator.

Inference 1 When $r>0$, If the non-negative matrix $\mathrm{A}$ satisfies the condition

$$
A^{-r}\left(X^{(0)}\right)^{T}>0, X^{(0)} D^{-r}=A^{-r}\left(X^{(0)}\right)^{T}
$$

is the reinforcement (weakening) buffer operator, then

$$
A^{r}\left(X^{(0)}\right)^{T}>0, X^{(0)} D^{r}=A^{r}\left(X^{(0)}\right)^{T}
$$

is a weakening (reinforcement) buffer operator.

The mathematical expressions of the positive real order weakening buffer operator and the positive real order weakening buffer sequence are as following.

Definition 1 The nonnegative observation data sequence is $X^{(0)}=\left(x^{(0)}(1), x^{(0)}(2), \cdots, x^{(0)}(n)\right)$.

$X^{(1)}=\left(x^{(1)}(1), x^{(1)}(2), \cdots, x^{(1)}(n)\right)$ is called the 1 order weakening buffer sequence, where

$$
x^{(1)}(k)=\frac{1}{n-k+1} \sum_{i=k}^{n} x^{(0)}(i), \quad k=1,2, \cdots, n .
$$

Definition 2 consider the nonnegative observation data sequence: $X^{(0)}=\left(x^{(0)}(1), x^{(0)}(2), \cdots, x^{(0)}(n)\right)$.

$X^{(r)}=\left(x^{(r)}(1), x^{(r)}(2), \cdots, x^{(r)}(n)\right)$ is called the $r, r \in N^{+}$ order weakening buffer sequence, where

$$
\begin{aligned}
& x^{(r)}(k)=\frac{1}{\sum_{i=k}^{n} C_{i-k+r-1}^{i-k}} \sum_{i=k}^{n} C_{i-k+r-1}^{i-k} x^{(0)}(i) \\
& k=1,2 \cdots, n, C_{r-1}^{0}=1, C_{n-1}^{n}=1 ; \\
& \quad r=0,1,2, \cdots, n \cdot \text { and } C_{r-1}^{0}=0, C_{n-1}^{n}=0 ; \\
& C_{i-k+r-1}^{i-k}=\frac{(i-k+r-1)(i-k+r-2) \cdots(r+1) r}{(i-k) !} .
\end{aligned}
$$

According to the properties of the Gamma function [6]:

$$
\begin{aligned}
& \Gamma(x)=\int_{0}^{\infty} e^{-t} t^{x-1} d t \\
& \Gamma(x+1)=x \Gamma(x)
\end{aligned}
$$

the combinatorial number formula can be transformed into:

$$
\begin{aligned}
C_{i-k+r-1}^{i-k} & =\frac{\Gamma(i-k+r-1+1)}{\Gamma(i-k+1) \Gamma(i-k+r-1+1)} \\
C_{i-k+r-1}^{i-k} & =C_{i-k+r-1}^{r-1} .
\end{aligned}
$$

The concept of positive real order is introduced.

Definition 3 The nonnegative observation data Sequence $X^{(0)}=\left(x^{(0)}(1), x^{(0)}(2), \cdots, x^{(0)}(n)\right)$.

The positive real order weakening buffer sequence is as follows:

$X^{(r)}=\left(x^{(r)}(1), x^{(r)}(2), \cdots, x^{(r)}(n)\right)$, where

$$
\begin{aligned}
& X^{(r)}=\left(\frac{1}{\sum_{i=1}^{n} C_{i-1+r-1}^{r-1}} \sum_{i=1}^{n} C_{i-1+r-1}^{r-1} x^{(0)}(i), \frac{1}{\sum_{i=2}^{n} C_{i-2+r-1}^{r-1}} \sum_{i=2}^{n} C_{i-2+r-1}^{r-1} x^{(0)}(i), \cdots,\right. \\
& \left.\frac{1}{\sum_{i=k}^{n} C_{i-k+r-1}^{r-1}} \sum_{i=k}^{n} C_{i-k+r-1}^{r-1} x^{(0)}(i), \cdots, \frac{1}{\sum_{i=n}^{n} C_{i-n+r-1}^{r-1}} \sum_{i=n}^{n} C_{i-n+r-1}^{r-1} x^{(0)}(i)\right) .
\end{aligned}
$$

Use $\sum_{k}^{r}$ to mark $C_{r+k-2}^{r-1}+C_{r+k-1}^{r-1}+\cdots+C_{r+n-2}^{r-1}$.

For the positive real order weakening buffer sequence, the matrix formula is as follows:

$$
X_{r}=\left[\begin{array}{ccccc}
\frac{1}{\Sigma_{1}^{r}} & \frac{C_{r}^{r-1}}{\Sigma_{1}^{r}} & \frac{C_{r+1}^{r-1}}{\Sigma_{1}^{r}} & \cdots & \frac{C_{r+n-2}^{r-1}}{\Sigma_{1}^{r}} \\
0 & \frac{C_{r}^{r-1}}{\Sigma_{2}^{r}} & \frac{C_{r+1}^{r-1}}{\Sigma_{2}^{r}} & \cdots & \frac{C_{r+n-2}^{r-1}}{\Sigma_{2}^{r}} \\
\cdots & \cdots & \cdots & \cdot & \vdots \\
0 & 0 & 0 & \cdots & \frac{C_{r+n-2}^{r-1}}{\Sigma_{n}^{r}}
\end{array}\right]\left[\begin{array}{c}
x^{(0)}(1) \\
x^{(0)}(2) \\
\vdots \\
x^{(0)}(n)
\end{array}\right]
$$




$$
A=\left[\begin{array}{ccccc}
\frac{1}{\Sigma_{1}^{r}} & \frac{C_{r}^{r-1}}{\Sigma_{1}^{r}} & \frac{C_{r+1}^{r-1}}{\Sigma_{1}^{r}} & \cdots & \frac{C_{r+n-2}^{r-1}}{\Sigma_{1}^{r}} \\
0 & \frac{C_{r}^{r-1}}{\Sigma_{2}^{r}} & \frac{C_{r+1}^{r-1}}{\Sigma_{2}^{r}} & \cdots & \frac{C_{r+n-2}^{r-1}}{\Sigma_{2}^{r}} \\
\cdots & \cdots & \cdots & . & \vdots \\
0 & 0 & 0 & \cdots & \frac{C_{r+n-2}^{r-1}}{\Sigma_{n}^{r}}
\end{array}\right] A \text { is the matrix }
$$

form of the positive real order weakening buffer operator $D$.

\section{Quadratic Time-Varying Parameters Discrete Grey Model (QTDGM)}

For the actual application of the data, it is distorted by interference from many external shock factors. In order to accurately mine the law of things. In this paper, a quadratic time-varying parameters discrete grey model based on positive real order weakening buffer operator is proposed.

Let the nonnegative observation data sequence be $X^{(0)}=\left\{x^{(0)}(1), x^{(0)}(2), \cdots, x^{(0)}(n)\right\}, \quad$ and $\quad$ its $\quad$ cumulative sequence is $X^{(1)}=\left\{x^{(1)}(1), x^{(1)}(2), \cdots, x^{(1)}(n)\right\}$,

$$
x^{(0)}(k+1) d_{1}=\left(\beta_{1}+\beta_{2} k+\beta_{3} k^{2}\right) x^{(0)}(k) d_{1}+\beta_{4} k^{2}+\beta_{5} k+\beta_{6}
$$

be the quadratic time-varying parameters discrete grey model (QTDGM), where $x^{(0)}(k)$ is the original sequence observation and $d_{1}$ is the positive real order weakening buffer operator.

Theorem 2 For QTDGM

$$
x^{(0)}(k+1) d_{1}=\left(\beta_{1}+\beta_{2} k+\beta_{3} k^{2}\right) x^{(0)}(k) d_{1}+\beta_{4} k^{2}+\beta_{5} k+\beta_{6}
$$

The least squares parameter estimates for this model are:

$$
\begin{aligned}
& \hat{\beta}_{1}=\frac{B_{1}}{A}, \hat{\beta}_{2}=\frac{B_{2}}{A}, \hat{\beta}_{3}=\frac{B_{3}}{A}, \\
& \hat{\beta}_{4}=\frac{B_{4}}{A}, \hat{\beta}_{5}=\frac{B_{5}}{A}, \hat{\beta}_{6}=\frac{B_{6}}{A} .
\end{aligned}
$$

For the convenience, let:

$$
\begin{aligned}
& M=\sum_{k=1}^{n-1}\left(x^{(0)}(k) d_{1}\right)^{2}, N=\sum_{k=1}^{n-1} k\left(x^{(0)}(k) d_{1}\right)^{2} ; C=\sum_{k=1}^{n-1} 1, D=\sum_{k=1}^{n-1} k, E=\sum_{k=1}^{n-1} k^{2}, \\
& F=\sum_{k=1}^{n-1} k^{3}, G=\sum_{k=1}^{n-1} k^{4} ; T=\sum_{k=1}^{n-1} k^{2}\left(x^{(0)}(k) d_{1}\right)\left(x^{(0)}(k+1) d_{1}\right), U=\sum_{k=1}^{n-1} k^{2}\left(x^{(0)}(k+1) d_{1}\right) \\
& V=\sum_{k=1}^{n-1} k\left(x^{(0)}(k+1) d_{1}\right), W=\sum_{k=1}^{n-1}\left(x^{(0)}(k+1) d_{1}\right) . \\
& O=\sum_{k=1}^{n-1} k^{2}\left(x^{(0)}(k) d_{1}\right)^{2}, P=\sum_{k=1}^{n-1} k^{3}\left(x^{(0)}(k) d_{1}\right)^{2}, Q=\sum_{k=1}^{n-1} k^{4}\left(x^{(0)}(k) d_{1}\right)^{2} ;
\end{aligned}
$$




$$
\begin{aligned}
& R=\sum_{k=1}^{n-1}\left(x^{(0)}(k) d_{1}\right)\left(x^{(0)}(k+1) d_{1}\right), S=\sum_{k=1}^{n-1} k\left(x^{(0)}(k) d_{1}\right)\left(x^{(0)}(k+1) d_{1}\right) \\
& H=\sum_{k=1}^{n-1} x^{(0)}(k) d_{1}, I=\sum_{k=1}^{n-1} k x^{(0)}(k) d_{1}, J=\sum_{k=1}^{n-1} k^{2} x^{(0)}(k) d_{1} \\
& K=\sum_{k=1}^{n-1} k^{3} x^{(0)}(k) d_{1}, L=\sum_{k=1}^{n-1} k^{4} x^{(0)}(k) d_{1} .
\end{aligned}
$$

So there are:

$$
\begin{aligned}
A & =\left(\begin{array}{llllll}
M & N & O & J & I & H \\
N & O & P & K & J & I \\
O & P & Q & L & K & J \\
J & K & L & G & F & E \\
I & J & K & F & E & D \\
H & I & J & E & D & C
\end{array}\right), B_{1}=\left(\begin{array}{llllll}
R & N & O & J & I & H \\
S & O & P & K & J & I \\
T & P & Q & L & K & J \\
U & K & L & G & F & E \\
V & J & K & F & E & D \\
W & I & J & E & D & C
\end{array}\right), B_{2}=\left(\begin{array}{lllllll}
M & R & O & J & I & H \\
N & S & P & K & J & I \\
O & T & Q & L & K & J \\
J & U & L & G & F & E \\
I & V & K & F & E & D \\
H & W & J & E & D & C
\end{array}\right), \\
B_{3} & =\left(\begin{array}{llllll}
M & N & R & J & I & H \\
N & O & S & K & J & I \\
O & P & T & L & K & J \\
J & K & U & G & F & E \\
I & J & V & F & E & D \\
H & I & W & E & D & C
\end{array}\right), B_{4}=\left(\begin{array}{llllllll}
N & N & O & I & H \\
O & O & P & S & J & I \\
J & K & L & T & K & J \\
I & J & K & V & E & D \\
H & I & J & W & D & C
\end{array}\right), B_{5}=\left(\begin{array}{llllll}
M & O & J & R & H \\
N & O & P & K & S & I \\
O & P & Q & L & T & J \\
J & K & L & G & U & E \\
I & J & K & F & V & D \\
H & I & J & E & W & C
\end{array}\right), \\
B_{6} & =\left(\begin{array}{llllll}
M & N & O & J & I & R \\
N & O & P & K & J & S \\
O & P & Q & L & K & T \\
J & K & L & G & F & U \\
I & J & K & F & E & V \\
H & I & J & E & D & W
\end{array}\right) .
\end{aligned}
$$

QTDGM can be obtained by using the estimated parameters. Thus variables $x^{(0)}(k+1) d_{1}$ is predictable.

Definition 5 The parameter estimates of QTDGM are shown in theorem2. The recursive formula of the first cumulative simulation value of sequence $x^{(0)}$ is as follows:

$$
\hat{x}^{(0)}(k+1) d_{1}=\left(\hat{\beta}_{1}+\hat{\beta}_{2} k+\hat{\beta}_{3} k^{2}\right) x^{(0)}(k) d_{1}+\hat{\beta}_{4} k^{2}+\hat{\beta}_{5} k+\hat{\beta}_{6}
$$

The simulated value of reduction is:

$$
\hat{x}^{(0)}(k+1)=\alpha^{(1)} \hat{x}^{(1)}(k+1)=\hat{x}^{(1)}(k+1)-\hat{x}^{(1)}(k)
$$

\section{Model Properties}

Theorem 3 Let $x^{(0)}$ be a nonnegative observation data sequence, where $x^{(0)}(k)=e^{a k}, k=1,2, \cdots, \hat{x}^{(0)}$ is the predicted value of QTDGM. Therefore $\hat{x}^{(0)}(k)=e^{a k}, k=1,2, \cdots n$.

Proof Let $A, B_{1}, B_{2}, B_{3}, B_{4}, B_{5}, B_{6}$ is the intermediate parameter of QTDGM parameter estimation value. $x^{(0)}(k)=e^{a k}, k=1,2, \cdots$ so $x^{(1)}(k+1)=e^{a} x^{(1)}(k)+e^{a}, k=1,2, \cdots$.

According to theorem 2, 


$$
\begin{aligned}
& B_{1}=\left(\begin{array}{llllll}
R & N & O & J & I & H \\
S & O & P & K & J & I \\
T & P & Q & L & K & J \\
U & K & L & G & F & E \\
V & J & K & F & E & D \\
W & I & J & E & D & C
\end{array}\right)=\left(\begin{array}{lllllll}
\sum_{k=1}^{n-1} x^{(1)}(k)\left(e^{a} x^{(1)}(k)+e^{a}\right) & N & O & J & I & H \\
\sum_{k=1}^{n-1} k x^{(1)}(k)\left(e^{a} x^{(1)}(k)+e^{a}\right) & O & P & K & J & I \\
\sum_{k=1}^{n-1} k^{2} x^{(1)}(k)\left(e^{a} x^{(1)}(k)+e^{a}\right) & P & Q & L & K & J \\
\sum_{k=1}^{n-1} k^{2}\left(e^{a} x^{(1)}(k)+e^{a}\right) & K & L & G & F & E \\
\sum_{k=1}^{n-1} k\left(e^{a} x^{(1)}(k)+e^{a}\right) & J & K & F & E & D \\
\sum_{k=1}^{n-1}\left(e^{a} x^{(1)}(k)+e^{a}\right) & I & J & E & D & C
\end{array}\right) \\
& =\left(\begin{array}{llllllll}
e^{a} M & N & O & J & I & H \\
e^{a} N & O & P & K & J & I \\
e^{a} O & P & Q & L & K & J \\
e^{a} J & K & L & G & F & E \\
e^{a} I & J & K & F & E & D \\
e^{a} H & I & J & E & D & C
\end{array}\right)+\left(\begin{array}{llllll}
e^{a} H & N & O & J & I & H \\
e^{a} I & O & P & K & J & I \\
e^{a} J & P & Q & L & K & J \\
e^{a} E & K & L & G & F & E \\
e^{a} D & J & K & F & E & D \\
e^{a} C & I & J & E & D & C
\end{array}\right)=e^{a} A \\
&
\end{aligned}
$$

Similarly, $B_{2}=0, B_{3}=0, B_{4}=0, B_{5}=0, B_{6}=e^{a} A$.

Therefore

$\hat{\beta}_{1}=e^{a} A, \hat{\beta}_{2}=0, \hat{\beta}_{3}=0, \hat{\beta}_{4}=0, \hat{\beta}_{5}=0, \hat{\beta}_{6}=e^{a} A$.

So, according to QTDGM and Definition 5,

$$
\begin{gathered}
\hat{x}^{(1)}(k+1)=e^{a} \hat{x}^{(1)}(k)+e^{a}=e^{a}+e^{2 a}+\cdots e^{(k+1) a} \\
\hat{x}^{(0)}(k+1)=e^{(k+1) a}, k=1,2, \cdots n
\end{gathered}
$$

Theorem 3 shows that the QTDGM has white exponential coincidence, so it has better simulation and prediction effect for high growth sequence.

Theorem 4 Let $x^{(0)}$ be a nonnegative observation data sequence, where $x^{(0)}(k)=a+k b, k=1,2, \cdots, \hat{x}^{(0)}$ is the predicted value of QTDGM, therefore $\hat{x}^{(0)}(k)=a+k b, k=1,2, \cdots n$.

Proof: process same as Theorem 3 .

Theorem 4 shows that the QTDGM can fully simulate linear sequences.

Theorem 5 Let $x^{(0)}$ be a nonnegative observation data sequence, where $x^{(0)}(k)=a+k b+k^{2} c, k=1,2, \cdots, \hat{x}^{(0)}$ is the predicted value of QTDGM, therefore $\hat{x}^{(0)}(k)=a+k b+k^{2} c, k=1,2, \cdots n$.

Proof: process same as Theorem 3.

Theorem 5 shows that QTDGM can completely simulate quadratic sequence.

Theorem 6 Let nonnegative observation data sequenceis the multiplicative transformation of $X^{(0)}$, where $y^{(0)}(k)=p x^{(0)}(k), p \geq 0$. The estimated values of their respective parameters obtained from QTDGM are $\beta_{x i}, \beta_{y i}, i=1,2, \cdots 6 \quad, \quad$ respectively. And $\beta_{x i}=\beta_{y i}, i=1,2,3 \cdots 6$.

Proof: According to theorem 2, it is obvious that it can be proved.

Theorem 7 Let nonnegative observation data sequence is the multiple transformation of $X^{(0)}$, where $y^{(0)}(k)=p x^{(0)}(k), p \geq 0$.

The estimated values of predicted value obtained from

QTDGM are $\hat{y}^{(0)}(k), \hat{y}^{(1)}(k), \hat{x}^{(0)}(k), \hat{x}^{(1)}(k)$, respectively.

And $\hat{y}^{(1)}(k)=p \hat{x}^{(1)}(k), \hat{y}^{(0)}(k)=p \hat{x}^{(0)}(k)$

Proof According to theorem 6, it is obvious that theorem 7 can be proved.

Theorem 6 and 7 describe the influence of multiple transformation on the parameters and simulation values of QTDGM, and prove that the model has consistency of scalable transformation. The multiple transformation of the original nonnegative observation data sequence does not affect the relative error of the simulation and prediction values of the model. In order to avoid the ill-posed problem of the model, when the observation value of the original sequence is large, the necessary processing is needed in advance $[7,8]$. 


\section{Model Base Value Iterative Optimization}

For the sake of analysis, section 5 discusses the selection iteration base value of the model. The model of the least squares fit is assumed to pass the point $\left(1, x^{(0)}(1)\right)$ in the plane coordinate system. When the modeling data $X^{(0)}$ is a special sequence such as exponential linearity, the hypothesis is valid. However, according to the principle of least squares, the fitting curve does not necessarily pass through point $\left(1, x^{(0)}(1)\right)$, so it is necessary to optimize the model iterative base value to avoid error accumulation and transmission [9, 10]. Consider adding a base value correction term to the definition 4 model to obtain the modified model as shown below:

$$
\begin{aligned}
& \hat{x}^{(0)}(k+1) d_{1}=\left(\hat{\beta}_{1}+\hat{\beta}_{2} k+\hat{\beta}_{3} k^{2}\right) x^{(0)}(k) d_{1}+\hat{\beta}_{4} k^{2}+\hat{\beta}_{5} k+\hat{\beta}_{6}, k=1,2,3, \cdots n-1 \\
& \hat{x}^{(0)}(1) d_{1}=x^{(0)}(1)+\varepsilon
\end{aligned}
$$

And $\hat{\beta}_{i}, i=1,2,3,4,5,6$ is obtained by theorem 2 . By iterating, the formula is changed as following:

$$
\begin{aligned}
& \hat{x}^{(0)}(1) d_{1}=x^{(0)}(1)+\varepsilon \\
& \hat{x}^{(0)}(2) d_{1}=\left(\hat{\beta}_{1}+\hat{\beta}_{2} k+\hat{\beta}_{3} k^{2}\right) x^{(0)}(1) d_{1}+\hat{\beta}_{4}+\hat{\beta}_{5}+\hat{\beta}_{6} \\
& \ldots \\
& \hat{x}^{(0)}(n) d_{1}=\left(\hat{\beta}_{1}+\hat{\beta}_{2}(n-1)+\hat{\beta}_{3}(n-1)^{2}\right) x^{(0)}(n-1) d_{1}+\hat{\beta}_{4}(n-1)^{2}+\hat{\beta}_{5}(n-1)+\hat{\beta}_{6}
\end{aligned}
$$

And $\hat{x}^{(0)}(k) d_{1}, k=1,2, \cdots n$ is a polynomial about $\mathcal{E}$. In order to minimize the error of simulation value, an optimization model is established:

$$
Q=\min \sum_{k=1}^{n}\left(x^{(0)}(k) d_{1}-\hat{x}^{(0)}(k) d_{1}\right)^{2}
$$

And $Q$ Is a quadratic polynomial about $\varepsilon$.

$\varepsilon^{2}$ coefficients are positive real numbers, according to , $\varepsilon$ can be obtained. Due to $\frac{d^{2} Q}{d \varepsilon^{2}}>0, \varepsilon$ is the minimum point of $Q$.

In summary, the modeling optimization and prediction process of QTDGM can be divided into the following steps:

Step1: Get the model parameter $\hat{\beta}_{i}, i=1,2, \cdots, 6$ value according to theorem 2;

Step2: Calculate $\hat{x}^{(0)}(k) d_{1}, k=1,2, \cdots n$ by formula (30).

$$
\text { And calculate } Q=\min \sum_{k=1}^{n}\left(x^{(0)}(k) d_{1}-\hat{x}^{(0)}(k) d_{1}\right)^{2}
$$

containing $\varepsilon$ by formula (30);

Step3: Solve the formula (31) optimization problem and get the iterative base value correction term $\varepsilon$;

Step4: Simulate and predict according to definition 5, formula (29) with the modified iterative base value;

Step5: Calculate the simulation error and test the model accuracy..

\section{Case Study: Comparison of Different Order Prediction Results}

Example 1: Taking Beijing annual science popularization fundraising as an example, the influence of positive real order weakening buffer operator of different orders on the prediction results of the model is compared. The raw data is shown in Table 1, and the data comes from the People's Republic of China National Bureau of statistics.

Table 1. Annual funding (unit: 10000RMB).

\begin{tabular}{llllllll}
\hline year & $\mathbf{2 0 0 8}$ & $\mathbf{2 0 0 9}$ & $\mathbf{2 0 1 0}$ & $\mathbf{2 0 1 1}$ & $\mathbf{2 0 1 2}$ & $\mathbf{2 0 1 3}$ & $\mathbf{2 0 1 4}$ \\
\hline funds & 134875.2 & 177933 & 204160 & 202819 & 221402 & 203614 & 217381 \\
\hline
\end{tabular}

Taking 2008-2014 annual funding for science popularization as the original sequence, the QTDGM of 0.6 , 0.8 and 1-order classical weakening buffer operator are established respectively. The data of 2015 are forecasted. The forecasting results and errors are shown in Table 2.

Table 2. Comparison errors.

\begin{tabular}{llll}
\hline order & $\mathbf{0 . 6}$ & $\mathbf{0 . 8}$ & $\mathbf{1}$ \\
\hline predicted value & 213338 & 214943 & 12701 \\
error & 716 & 2321 & -225323 \\
Relative error & $0.34 \%$ & $1.09 \%$ & $105.97 \%$ \\
\hline
\end{tabular}

Comparing with the results in Table 2, the 0.6 order weakening buffer operator can realize fine-tuning of the model, and has higher quasi-precision. It can better mine the development trend of the system and get better prediction accuracy.

EXAMPLE 2: In order to verify the validity of the model proposed in this paper, the raw coal output data of China from October 2017 to May 2018 are used to simulate and predict, and the data comes from the China Statistical Yearbook. 
Table 3. Raw coal production (unit: 10000 ton).

\begin{tabular}{lllllllll}
\hline Month & $\mathbf{2 0 1 7 . 1 0}$ & $\mathbf{1 1}$ & $\mathbf{1 2}$ & $\mathbf{2 0 1 8 . 1}$ & $\mathbf{2}$ & $\mathbf{3}$ & $\mathbf{4}$ & $\mathbf{5}$ \\
\hline Values & 28354.3 & 29997.5 & 31487.2 & 29021.8 & 29329.7 & 29699 & 29801.7 & 28150.4 \\
\hline
\end{tabular}

The result is predicted by simulation. When the order is 0.6 , the output forecast for May is 30167.4. The relative error is $7.2 \%$. When the order is 0.8 , the output forecast for May is 30137.1 The relative error is $7.0 \%$. When the accumulator is used, the prediction effect is particularly poor. The prediction result is 1174, and the relative error is over $90 \%$.

Example 3: Taking the special funds for science popularization in Jiangsu Province as an example to compare the effects of positive real order weakening buffer operator with different orders on the prediction results. The original data are shown in Table 4, the statistical yearbook of China.

Table 4. Annual average funding (unit: 10000RMB).

\begin{tabular}{lllllllll}
\hline year & 2008 & 2009 & 2010 & 2011 & 2012 & 2013 & 2014 & 2015
\end{tabular}

\begin{tabular}{llllllllll}
\hline funds & 45787 & 64579 & 72688 & 84824 & 93538 & 91378 & 103743 & 104307 \\
\hline
\end{tabular}

Taking the annual fund raising of science popularization in Jiangsu Province from 2008 to 2014 as the original sequence, the linear time-varying parameter discrete models of $0.3,0.6$ and 0.8 order weakening buffer operator are also established. The data of 2015 are forecasted. The forecasting results and errors are shown in Table 5.

Table 5. Comparison errors.

\begin{tabular}{llll}
\hline order & $\mathbf{0 . 3}$ & $\mathbf{0 . 6}$ & $\mathbf{0 . 8}$ \\
\hline predicted value & 98377 & 92231 & 91343 \\
error & -5970 & -12075 & -12963 \\
Relative error & 5.72 & $11.57 \%$ & $12.48 \%$ \\
\hline
\end{tabular}

Comparing with the results in Table 4, the 0.3 order weakening buffer operator can realize fine-tuning of the model, with higher quasi-precision, better development trend of the mining system and better prediction accuracy.

In order to further explore the prediction accuracy of the model, this paper quotes the linear time-varying grey discrete prediction model and compares it with the model in this paper. The data used in case 2 shows the prediction results in Table6.

Table 6. Forecast result

\begin{tabular}{lllllll}
\hline QTDGM & & & TGDM & \\
\hline order & 0.3 & 0.8 & 1 & & 0.3 & 0.8 \\
predicted value & 98377 & 92231 & 91343 & predicted value & 54592 & 95200 \\
error & -5970 & -12075 & -12963 & error & -49715 & -9107 \\
\hline
\end{tabular}

Under the optimum order of 0.3 and 0.8 , the quadratic linear time-varying grey discrete forecasting model has better forecasting effect. In order to further evaluate the prediction effect, the following two prediction accuracy criteria are used to evaluate the results as shown in Table 7 ..

$$
\begin{aligned}
& \text { MAPE: } \frac{1}{n} \sum_{k=1}^{n}\left|\frac{\hat{x}(k)-x(k)}{x(k)}\right| \\
& \text { AME: } \frac{1}{n} \sum_{k=1}^{n}|\hat{x}(k)-x(k)|
\end{aligned}
$$

Table 7. Error comparison.

\begin{tabular}{lll}
\hline & QTDGM & TDGM \\
\hline MAPE & $9.91 \%$ & $30.81 \%$ \\
AME & 10336 & 32142 \\
\hline
\end{tabular}

From the results of Table 7, it can be concluded that the error of the QTDGM is less than that of the TGDM. Therefore, according to the analysis of the above two examples, it can be concluded that the prediction accuracy of the QTGDM is higher, and the prediction effect is indeed better than that of the TGDM and the traditional integer-order model, which can get more accurate simulation and prediction results..

\section{Conclusion}

The discrete grey model with quadratic time-varying parameters constructed in this paper has changed the problem of constant growth rate of simulation value of the original discrete grey model. The quadratic time term is introduced and the iterative optimization method of the model base value is given. Compared with the original time-varying grey discrete prediction model, more accurate prediction and parameter estimation are achieved.

By useing the positive real order weakening buffer operator, fine tune the intensity of the accumulation to precision prediction. After three examples, the operator makes the prediction result more close to the real value. It can be applied to precise prediction of small samples.

\section{References}

[1] Ju-Long D. Control problem of grey systems [J]. Systems \& Control Letters, 1982, 1(5): 288-294.

[2] Deng J L. The grey exponential law for accumulative generation - the problem of optimal processing of information in grey control systems. [J]. J. huazhong Univ.sci.tech, 1987(5): 7-12. 
[3] Li-Yun W U, Zheng-Peng W U, Mei L I, et al. Quadratic time-varying parameters discrete grey model $[\mathrm{J}]$. Systems Engineering-Theory \& Practice, 2013, 33(11): 2887-2893.

[4] Pan X J, Wei Z, Tian Z, et al. Fractional Order Discrete Grey Model and Its Application in Spare Parts Demand Forecasting [J]. Acta Armamentarii, 2017, 38(4): 785-792.

[5] $\mathrm{Wu} \mathrm{L}$, Liu S, Yao L, et al. Grey system model with the fractional order accumulation [J]. Communications in Nonlinear Science \& Numerical Simulation, 2013, 18(7): 1775-1785.

[6] Laurinčikas A, Garunkštis R. Euler Gamma-Function [M]// The Lerch Zeta-function. Springer Netherlands, 2003: 1-15.

[7] Wu L, Qi Y, Wu Z. Grey Discrete time-varying Model and Its application $[\mathrm{C}] / /$ IEEE International Conference on Grey Systems and Intelligent Services. IEEE, 2015: 242-246.

[8] Xie N, Liu S. Research on discrete grey model and its mechanism [C]// IEEE International Conference on Systems, Man and Cybernetics. IEEE, 2006: 606-610 Vol. 1.

[9] Zhang S J, Chen S Y, Transportation S O, et al. Optimization of GM $(1,1)$ Power Model and Its Application [J]. Systems Engineering, 2016.
[10] Xie N M, Liu S F. Discrete grey forecasting model and its optimization [J]. Applied Mathematical Modeling, 2009, 32(2): 1173-1186.

\section{Biography}

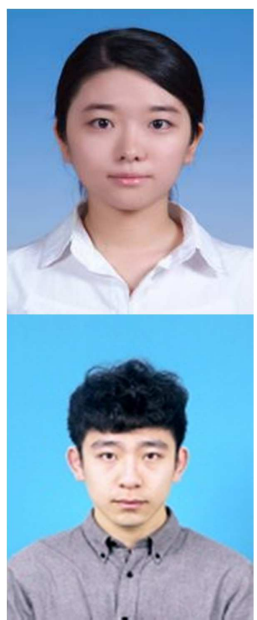

Mengdi Jin was born in 1994. She received B. S. degree in information and computing science from Communication University of China. Currently she is a master degree candidate of Communication University of China. Her research interests include grey system and econometrics.

Jiwei Liu was born in 1994. He received B. S. degree in mathematics and applied mathematics from Qufu Normal University. Currently he is a master degree candidate of Communication University of China. His research interests include grey system and econometrics. 